\title{
INHIBICIÓN DE LA BUTIRILCOLINESTERASA EN EL GORRIÓN CASERO EXPUESTO A MALATIÓN
}

\author{
Butyrylcholinesterase inhibition in the house sparrow exposed to malation
}

\author{
Aixa Najla BUJDUD LEÓN ${ }^{1}$, Martín Emilio PEREDA SOLÍS ${ }^{2 *}$, \\ José Hugo MARTÍNEZ GUERRERO ${ }^{2}$ y Maribel GUERRERO CERVANTES ${ }^{2}$
}

${ }^{1}$ Programa Interinstitucional de Maestría en Ciencias Agropecuarias y Forestales, Facultad de Medicina Veterinaria y Zootecnia, Universidad Juárez del Estado de Durango, km 11.5 Carretera Durango - Mezquital, Durango, Dgo. C.P. 34307, México

${ }^{2}$ Facultad de Medicina Veterinaria y Zootecnia, Universidad Juárez del Estado de Durango, km 11.5 Carretera Durango - Mezquital, Durango, Dgo. C.P. 34307, México

*Autor para correspondencia: mepereda@ujed.mx

(Recibido: mayo 2017; aceptado: febrero 2019)

Palabras clave: toxicidad, plaguicida, organofosforados, acetilcolina, Passer domesticus

\section{RESUMEN}

Se estimó el efecto del malatión (insecticida organofosforado) en individuos de gorrión casero (Passer domesticus) capturados en una zona agrícola del municipio de Durango, México. La butirilcolinesterasa se inhibe con los insecticidas organofosforados, por ello mediante espectrofotometría se determinó su actividad previa y posterior al consumo de alimento tratado con malatión en 19 organismos. Los resultados muestran una disminución de la actividad $\mathrm{BChE}(11.58 \%)$ posterior al tratamiento $(\mathrm{p}=0.03)$. Se exploró la posible relación entre el peso de las aves y la actividad $\mathrm{BChE}$ antes y después de la exposición al insecticida organofosforado pero no se encontró correlación entre estas dos variables (Pearson, $r=0.114$ ), por lo que se decidió no utilizar la variable peso como covariable en los análisis para comparar la actividad enzimática de las aves. El peso de las aves disminuyó en promedio $1.54 \mathrm{~g}$ posterior a la exposición, posiblemente debido al estrés de captura y la falta de adaptación al cautiverio. Es posible que el gorrión casero y otras aves que cohabitan en las zonas agrícolas donde se aplica malatión, así como otros insecticidas organofosforados, sean propensos a desarrollar diferentes niveles de intoxicación y que en algunos casos se comprometa su estado de salud.

Key words: toxicity, pesticide, organophosphorus, acetylcholine, Passer domesticus

\begin{abstract}
The effect of malathion (organophosphate insecticide) on individuals of the house sparrow (Passer domesticus) caught in an agricultural area of the municipality of Durango, Mexico, was estimated. Butyrylcholinesterase is inhibited by organophosphorus insecticides, therefore, its previous and subsequent activity to the consumption of food treated with malathion was determined in 19 organisms by spectrophotometry. The results show a decrease in BChE activity $(11.58 \%)$ after treatment $(\mathrm{p}=0.03)$. The possible relationship between the weight of the birds and $\mathrm{BChE}$ activity before and
\end{abstract}


after exposure to the organophosphorus insecticide was explored but no correlation was found between these two variables (Pearson, $r=0.114$ ), so it was decided not to use the variable weight as a covariate in the analyses to compare the enzymatic activity of the birds. The weight of the birds decreased on average $1.54 \mathrm{~g}$ after the exposure, possibly due to the stress of capture and the lack of adaptation to captivity. It is possible that the house sparrow and other birds that co-inhabit the agricultural areas where malathion is applied, as well as other organophosphorus insecticides, are prone to develop different levels of intoxication and that in some cases their health condition is compromised.

\section{INTRODUCCIÓN}

Debido al aumento poblacional y la consecuente demanda de alimentos, cada día una mayor superficie del planeta se dedica a la práctica de la agricultura, lo cual conlleva a la transformación de suelos de su vocación original (bosques, selvas y pastizales) a campos agrícolas (FAO 2000). Como es de esperarse, el cambio no evolutivo trae como consecuencia la alteración en el orden natural, ya que se modifica la diversidad biológica. Los cultivos favorecen la reproducción de diversos organismos (invertebrados y vertebrados) debido a que son fuente rica de alimento; bajo estas circunstancias estos organismos son denominados plaga, término que define a los organismos que interfieren con la conveniencia o bienestar del hombre u otra especie de su interés (Vega 1985), los cuales son combatidos con gran cantidad y variedad de plaguicidas. Tan sólo en los Estados Unidos de América, cada año se aplican más de 2 millones de toneladas de plaguicidas (Alavanja et al. 2004, Weiss et al. 2004), lo que conlleva a que potencialmente todos los organismos estén expuestos a partir de diferentes fuentes como alimentos, agua y suelo. Su toxicidad, así como el mal uso que se hace de ellos, genera riesgos para la conservación de la biodiversidad de los agroecosistemas (Bernardos y Zaccagnini 2008).

Los organofosforados (OF) son los plaguicidas sintéticos más utilizados en la agricultura, son de amplio espectro y afectan el sistema nervioso mediante la inhibición de las colinesterasas (acetilcolinesterasa [AChE o colinesterasa eritrocitaria] y butirilcolinesterasa [BChE o colinesterasa plasmática]), las cuales transforman el neurotransmisor acetilcolina (ACh) en colina y acetato. En el sistema nervioso central (SNC), la ACh está particularmente implicada en los circuitos de la memoria, la recompensa ("reward"), los circuitos extra piramidales y en el sistema nervioso periférico (SNP) específicamente en el sistema nervioso autónomo (Flores y Segura 2005). La acumulación de Ach induce la estimulación continua de los músculos, glándulas y del SNC, causando hiperactividad nerviosa que finaliza con la muerte del individuo (Costa 2006).

Los organofosforados son altamente tóxicos y se absorben rápidamente por las vías respiratoria y dérmica, así como también por la ingestión oral (Jeyaratnam y Maroni 1994); son solubles en agua; se infiltran hasta alcanzar las aguas subterráneas, son absorbidos por las plantas, transferidos a las hojas y tallos donde quedan al abasto de insectos que comen hojas o se alimentan de savia (Vega 1985). Con el paso del tiempo los OF son degradados por las bacterias presentes en el suelo, hojas y superficie de los frutos, así como por los sistemas enzimáticos propios de las plantas mediante la hidrólisis con la pérdida del grupo amino, S-metil y el grupo O-metil (Palacios y Campos 2010). Estudios sobre la bioacumulación de los OF en los cultivos de tomate han reportado una vida media en el fruto de 4.8 a 5.1 días y en hojas de 5.5 a 5.9 días, por lo que si no se toman en cuenta estas consideraciones, los OF pueden estar presentes en algunos alimentos en el mercado como lo documentan los estudios exploratorios realizados en Colombia y Perú (Murcia y Stashenko 2008, Palacios y Campos 2010).

Un nivel disminuido de la $\mathrm{BChE}$ y la $\mathrm{AChE}$ en el tejido animal es un signo de que se ha producido algún tipo de exposición a un agente inhibidor de estas enzimas, por lo que la determinación de la actividad $\mathrm{BChE}$ es un buen biomarcador de exposición o daño a este tipo de agentes, ya que es un método simple, rápido y económico (Hatch 1988, Arveláez y Eblen-Zajjur 2013).

En aves, la principal vía de intoxicación por OF es el consumo de alimentos contaminados, si bien también puede ocurrir por inhalación al sobrevolar o habitar los campos de cultivo y sus inmediaciones (Cobos et al. 2006), provocando reducción en el consumo de alimento, cambios en la conducta de vuelo y postura (Varea 2003, Cobos et al. 2006). Estudios similares han reportado salivación, fasciculación, letargia, anorexia, efectos en la reproducción y muerte (Grue et al. 1981, Dabbert et al. 1996, Burkepile et al. 2002). En el caso de que los organismos no mueran, 
los signos por lo general disminuyen días después de la exposición, cuando los niveles de acetilcolina vuelven a la normalidad (Sarwar 2015).

Las respuestas metabólicas a OF son similares entre aves y mamíferos, sin embargo las primeras son más sensibles que los últimos debido a la menor actividad hepática y a la actividad de la A-esterasa (Hill 1994, Lari et al. 1994). En los mamíferos la vida media de los OF y sus productos de biotransformación es relativamente corta (alrededor de $48 \mathrm{~h}$ ), su metabolismo se lleva a cabo mediante oxidasas, hidrolasas y glutatión-S-transferasas; su eliminación es rápida y tiene lugar por la orina, en menor cantidad en heces y aire expirado, su máxima excreción se alcanza a los dos días, posteriormente disminuye rápidamente (Palacios y Campos 2010). En el entendido de que los OF representan un riesgo a la salud de la avifauna, este trabajo plantea evaluar y comparar los niveles de butirilcolinesterasa en gorriones domésticos en respuesta a su exposición al malatión, un plaguicida organofosforado utilizado en la agricultura.

\section{MATERIAL Y MÉTODOS}

\section{Sitio de estudio}

El presente trabajo se realizó en la Facultad de Medicina Veterinaria y Zootecnia (FMVZ) de la Universidad Juárez del Estado de Durango (UJED) con las coordenadas geográficas N 23⒌ $57^{\prime} 21.535^{\circ}$ " y O 104³4'24.419" (Fig. 1).

\section{Método de colecta}

Para la captura de aves se contó con la autorización SEMARNAT-SPGA/DGVS/10768/16M, se utilizó una red de niebla fabricada de poliéster negro modelo KTX de Avian Research Supplies, de $36 \mathrm{~mm}$ de malla, $2.6 \mathrm{~m}$ de alto por $12 \mathrm{~m}$ de largo y cuatro bolsas. La captura y procesamiento de las aves se realizó entre las 10:00 y 12:00 h, la identificación se realizó marcando los tarsos de las aves con cinco colores diferentes de pintura esmalte, asignándole a cada color un número.

\section{Toma de muestras}

A cada ave se le tomó una muestra de sangre mediante punción de la vena braquial (ala) con un tubo capilar heparinizado, obteniendo un máximo de 100 microlitros por cada ave (menos del $2 \%$ del peso vivo). Las muestras de sangre se colocaron en tubos de vidrio marcados con los datos del ave, sellados y colocados en hielo, para su posterior transporte al laboratorio de posgrado de la FMVZ UJED.

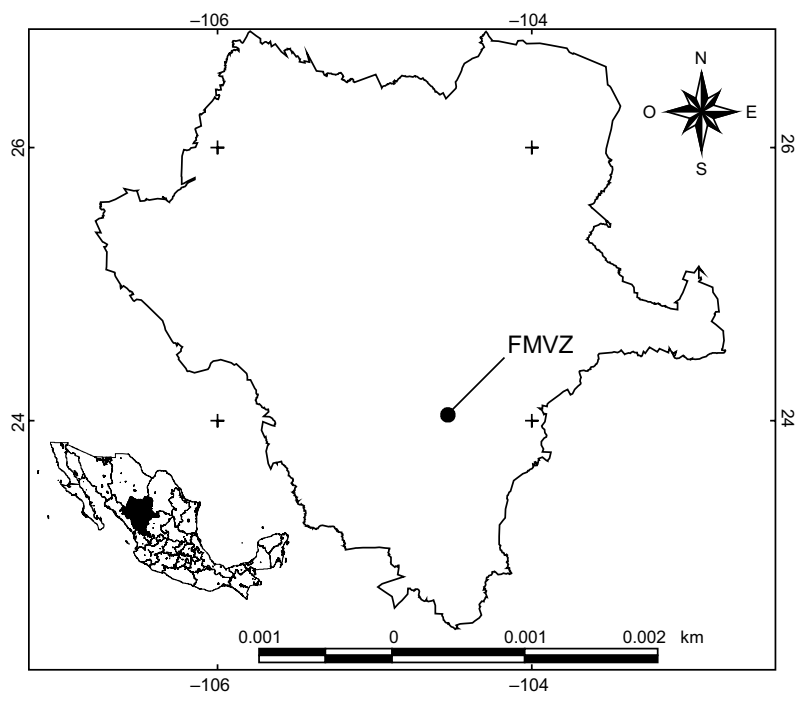

Fig. 1. Localización del sitio de estudio.

Para cada ave capturada se determinó su peso mediante una báscula Ohaus ${ }^{\circledR}$ (Mod. CS 2000, capacidad de 2000 g y 0.1 g de precisión). Posteriormente, las aves capturadas se alojaron en cuatro jaulas (distribuidas 5-5-5-4 aves por jaula) con malla mosquitera, en donde se les ofreció agua y alimento (alpiste) durante cinco días a modo de adaptación. Al sexto día se les ofreció el alimento tratado mediante aspersión con malatión [dietil (dimetoxitiofosfori(tio)) succinato], este $\mathrm{OF}$ se administró en una botella de plástico de $100 \mathrm{ml}$ con atomizador donde se preparó una solución al $0.5 \%$ siguiendo la recomendación del fabricante (1 L de insecticida por $200 \mathrm{~L}$ de agua/ha). Posteriormente la solución se roció sobre semillas de alpiste esparcidas en una capa delgada sobre una charola. El total de alimento preparado fue $675 \mathrm{~g}$, cantidad que se estimó para proveer de alimento de manera suficiente a las aves durante el tiempo de exposición. Al séptimo día se realizó la segunda toma de muestras y pesaje; posteriormente las aves se liberaron en el mismo lugar de captura.

\section{Procesamiento de las muestras}

La medición de la actividad de la $\mathrm{BChE}$ se realizó mediante el método de Ellman et al. (1961) modificado por Hill y Flemming (1982), para esto se utilizó un equipo de la marca Spinreact ${ }^{\circledR}$, el cual trabaja con amortiguador de fosfatos y butiriltiocolina como substrato. Las lecturas se hicieron con un espectrofotómetro (405 nm) modelo $10 \mathrm{~S}$ UV-VIS de la marca Genesys ${ }^{\circledR}$. La determinación de la BChE plasmática tiene la ventaja de que el 
sustrato utilizado, butiriltiocolina, es más estable que la acetiltiocolina respecto a la temperatura y $\mathrm{pH}$ (Whittaker 1974).

Se utilizaron los programas estadísticos NCSS (Hintze J., Kaysville, UT, EUA) y SAS (SAS Institute, Cary, NC, EUA) para el análisis de los datos. Para explorar la posible relación entre las variables actividad de la BChE, sexo y peso se utilizó un análisis de covarianza. Se utilizó la prueba $t$ de Student $(\alpha=0.05)$ para comparar los niveles de butirilcolinesterasa antes y después de la exposición al malatión.

\section{RESULTADOS}

Se capturaron 19 individuos de gorrión casero, 15 machos y cuatro hembras. Se exploró la posible relación entre el peso de las aves y la actividad enzimática de la $\mathrm{BChE}$ antes y después de la exposición al insecticida organofosforado pero no se encontró correlación entre estas dos variables (Pearson, $r=$ 0.114), por lo que se decidió no utilizar la variable peso como covariable en los análisis para comparar la actividad enzimática de las aves. Al comparar los pesos de las aves antes y después del consumo de alimento con insecticida (Cuadro I) se observó disminución general $(\mathrm{p}=0.007)$ de $1.54 \mathrm{~g}$. El peso corporal del grupo de hembras fue similar $(\mathrm{p}=0.057)$ $\mathrm{y}$ en los machos se observó disminución $(\mathrm{p}=0.025)$ de $1.58 \mathrm{~g}$.

CUADRO I. PESO PROMEDIO (g) DEL GORRIÓN CASERO, ANTES (T1) Y DESPUÉS (T2) DEL CONSUMO DE ALIMENTO TRATADO CON MALATIÓN

\begin{tabular}{llcr}
\hline & $\mathrm{n}$ & \multicolumn{1}{c}{ T1 } & \multicolumn{1}{c}{ T2 } \\
\hline Total & 19 & $26.44 \pm 1.54$ & $24.9 \pm 1.78$ \\
Hembras & 4 & $25.87 \pm 0.76$ & $24.47 \pm 0.87$ \\
Machos & 15 & $26.59 \pm 1.68$ & $25.01 \pm 1.96$ \\
\hline
\end{tabular}

*Promedio \pm desviación estándar

El nivel de colinesterasa plasmática promedio del grupo de 19 aves tras un día de exposición presentó una reducción significativa $(\mathrm{p}=0.03)$ de $11.58 \%$ (Cuadro II, Fig. 2). Sin embargo, no se observaron diferencias estadísticas cuando se desagregaron por sexo (hembras $\mathrm{p}=0.39, \mathrm{~F}=0.85$, g.1. $=1 \mathrm{y}$ machos $\mathrm{p}=0.06, \mathrm{~F}=3.71$, g.l. $=1$ ) a pesar que los valores promedio fueron menores.

\section{DISCUSIÓN}

Fossi et al. (1996), Cordi et al. (1997) y Cobos et al. (2006) reportan una relación positiva entre el peso y los niveles de colinesterasa en plasma, sin embargo a diferencia de éstos, en el presente estudio no se encontró una correlación entre el peso y los niveles de butirilcolinesterasa, por lo que no utilizamos el peso como una covariable para analizar las diferencias de la actividad enzimática de la $\mathrm{BCHe}$ de las aves.

Contreras y Ubilla (2013) mencionan que los estímulos nocivos tales como exposición a ruidos, sustrato, temperatura, proximidad a humanos, aglomeraciones, grupos sociales anormales causan la pérdida de peso y disminución de la condición corporal de las aves en cautiverio, por tanto es posible que la disminución del peso en las aves pudiera deberse al estrés de captura y la falta de adaptación en cautiverio (siete días).

Ninguno de los ejemplares expuestos al malatión mostró signos de intoxicación como reducción del consumo de alimento, salivación, fasciculación, letargia, anorexia o muerte. Según Varea y colaboradores (2003) la disminución de la actividad de colinesterasa plasmática de un $30 \%$ a $50 \%$ en relación con los valores normales indica exposición, aunque los síntomas pueden no aparecer hasta que las cifras de esta enzima se han reducido a un $20 \%$ de actividad o menos. Los datos obtenidos en nuestro trabajo muestran disminución general de $11.58 \%$ a partir de los valores de referencia obtenidos en el análisis previo a la exposición (T1), lo cual apoya la hipótesis de que el consumo vía oral de alimento contaminado con insecticidas organofosforados provoca una inhibición de la BChE plasmática (Maia et al. 2012, Palacios y Campos 2010).

La BChE está presente en forma soluble en casi todos los tejidos, principalmente en hígado y plasma, pero en poca concentración en el sistema nervioso central y periférico, esta enzima es de utilidad en la evaluación de exposición crónica a OF (Palacios y Campos 2010).

La actividad de la $\mathrm{BChE}$ plasmática se inhibe más rápido que la de la colinesterasa eritrocitaria. De la misma forma, su recuperación es más rápida debido a la síntesis hepática, mientras que la recuperación de la actividad eritrocitaria depende de la lenta producción de nuevas células (Varea et al. 2003).

En un estudio con halcones cola roja (Buteo jamaicensis) donde midieron la ChE plasmática y metabolitos de compuestos OF en el tracto gastrointestinal, encontraron que tanto la vía de exposición al 
CUADRO II. CUANTIFICACIÓN DE LA ACTIVIDAD DE BUTIRILCOLINESTERASA (UNIDADES INTERNACIONALES / L) EN GORRIÓN CASERO TRATADO CON MALATIÓN

\begin{tabular}{lcccc}
\hline Sexo & T1* & T2* & $\mathrm{p}^{* *}$ & Inhibición $\%$ \\
\hline Total & $299.02 \pm 60.84$ & $264.37 \pm 35.63$ & 0.03 & 11.58 \\
Hembra & $294.66 \pm 38.36$ & $273.60 \pm 24.84$ & 0.39 & 7.14 \\
Macho & $300.18 \pm 66.66$ & $261.91 \pm 38.33$ & 0.06 & 12.74 \\
\hline
\end{tabular}

*Promedio \pm desviación estándar. T1 = previo al tratamiento, $\mathrm{T} 2$ = posterior al tratamiento, ${ }^{* *} \mathrm{p}<0.05$ indica diferencia estadística significativa entre tratamientos.

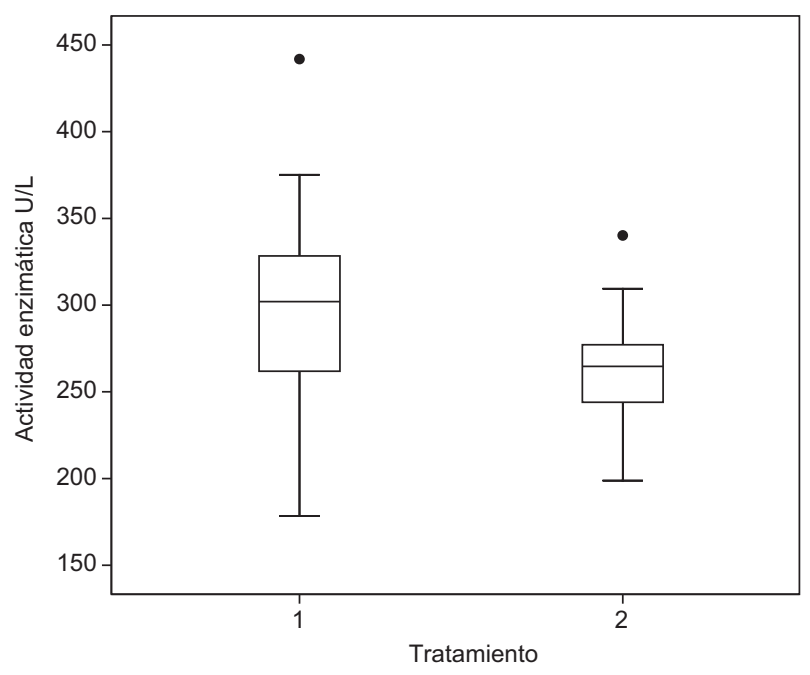

Fig. 2. Niveles medios de butirilcolinesterasa entre tratamientos, previo (1) y posterior (2) al consumo de alimento con malatión. En cada barra se representa el valor máximo, mediana, valor mínimo de cada tratamiento y valores atípicos $(\bullet)$.

insecticida (ingestión, inhalación y contacto capilar) como el tipo del mismo, son los que determinan el grado de toxicidad (Hooper et al. 1989).

Neithammer y Baskett (1983) comprobaron que la actividad enzimática varía de acuerdo a las especies, y que ésta presenta porcentajes de inhibición variables, por tanto no es de sorprender que los resultados del presente estudio varíen de los encontrados por Cobos et al. (2006), en donde la inhibición de la BChE en la especie Turdus grayi fue de $49.3 \%$ contra el $11.5 \%$ del presente estudio.

Aunado a lo anterior no se debe descartar el hecho que esta investigación se realizó con un solo día de exposición al insecticida, por tanto debe considerarse que los OF cuando se aplican al suelo y en plantas se absorben por hojas, tallos, corteza, raíces y circulan en la savia haciéndola tóxica (Ponce et al. 2006), es decir, que los niveles de BChE podrían disminuir en mayor proporción tras una alimentación con los frutos, semillas e inclusive insectos durante periodos prolongados.

Cabe señalar que para el caso del Valle del Guadiana en el estado de Durango, los insecticidas como paratión, diazinón y malatión han reducido su demanda (entrevista con distribuidores). Pese a lo anterior hace falta realizar más estudios en el estado de Durango para complementar los datos aquí obtenidos y tomar como práctica habitual el analizar estacionalmente el alimento destinado a consumo tanto animal como humano y de esta manera constatar si existe la posibilidad de intoxicaciones por consumo, recordando que en 2015 los insecticidas paratión, malatión y diazinón fueron clasificados como cancerígenos por la agencia internacional para investigación del cáncer (IARC 2015).

\section{CONCLUSIONES}

El gorrión casero mostró ser una especie sensible a la presencia de compuestos organofosforados. En este experimento el uso de las dosis recomendadas por los fabricantes para la aplicación de este compuesto disminuyó los niveles de butirilcolinesterasa plasmática; sin embargo, no resultó letal ni ocasionó signos de intoxicación en las aves expuestas. La concentración de butirilcolinesterasa plasmática se confirma como un biomarcador útil para medir el efecto de la exposición de las aves a compuestos organofosforados.

\section{AGRADECIMIENTOS}

Los autores agradecen a los editores y revisores sus valiosos comentarios y contribuciones a este manuscrito. También se agradece a Daniel Sierra Franco por la elaboración del mapa, a los jóvenes Samuel Arroyo Arroyo, Selene Guillen 
González y Fernando Flores Morales por su ayuda para la captura y manejo de las aves.

\section{REFERENCIAS}

Alavanja M.C., Hoppin J.A. y Kamel F. (2004). Health effect of chronic pesticide exposure: cancer and neurotoxicity. Annu. Rev. Public Health 25, 155-197. DOI: 10.1146/annurev.publhealth.25.101802.123020

Arveláez L. y Eblen-Zajjur A. (2013). Inhibición de butirilcolinesterasa por exposición a malatión: Efectos sobre nocicepción y motricidad en ratas. Salus 17 (2), 31-36.

Bernardos J.N. y Zaccagnini M.E. (2008). Evaluación del riesgo de toxicidad aguda para aves por uso de insecticidas en arroceras. En: Primer taller para la Conservación de Aves Playeras Migratorias en Arroceras del Cono Sur (V.M. De la Balze y D.E. Blanco, Eds.). Wetlands International, Buenos Aires, Argentina. pp. 1-4.

Burkepile N.A., Hewitt D.G., Waggerman G.L., Small M.F. y Hellgren E.C. (2002). Effects of methylparathion on white-winged dove productivity and reproductive behavior. J. Wildl. Manage. 66 (1), 202-211. DOI: $10.2307 / 3802886$

Cobos V.M., Mora M.A. y Escalona G. (2006). Inhibición de colinesterasa plasmática en el zorzal pardo (Turdus grayi), expuestos a diazinón en cultivos de papaya maradol en Yucatán, México. Rev. Toxicol. 23 (1), 17-21.

Contreras C.P. y Ubilla C. (2013). Evaluación del bienestar animal de aves rapaces en rehabilitación, descripción de técnicas que lo promuevan y mejoren su tasa de reintroducción. Av. Ciencias Veterinarias 28 (2), 1-12. DOI: 10.5354/0719-5273.2014.30201

Cordi B.C., Fossi M.C. y Depledge M. (1997). Temporal biomarker responses in wild passerine birds exposed to pesticide spray drift. Environ. Toxicol. Chem. 16 (10), 2118-2124.

Costa L.G. (2006). Current issues in organophosphate toxicology. Clin. Chim. Acta 33 (1-2), 1-13. DOI: 10.1016/j.cca.2005.10.008

Dabbert C.B., Sheffield S.R. y Lochmiller R.I. (1996). Northern bobwhite egg hatchability and chick immunocompetence following a field application of Diazinon. Bull. Environ. Contam. Toxicol. 56 (4), 612-616. DOI: $10.1007 / \mathrm{S} 001289900088$

Ellman G.L., Courtney K.D., Andres V. JR. y Featherstone R.M. (1961). A new and rapid colorimetric determination of acetylcholinesterase activity. Biochem. Pharmacol. 7, 88-95. DOI: 10.1016/0006-2952(61)90145-9

FAO (2000). Producción y productividad agrícola en los países en desarrollo. En: El estado mundial de la agricultura y la alimentación. (Organización de las Naciones Unidas para la Agricultura y la Alimentación, Ed.). FAO, Roma, Italia, pp. 243-285.

Flores M.E. y Segura J.E. (2005). Estructura y función de los receptores de acetilcolina de tipo muscarínico y nicotínico. Rev. Mex. Neuroci. 6 (4), 315-326.

Fossi M.C., Lari L. y Cassini C.S. (1996). Interspecies variation of " $B$ " esterase in birds: the influence of size and feeding habits. Arch. Environ. Contam. Toxicol. $31,525-532$.

DOI: $10.1007 / \mathrm{BF} 00212436$

Fossi M.C., Massi A. y Loenzio C. (1994). Blood esterase inhibition in birds as an index of organophosphorus contamination; field and laboratory studies. Ecotoxitology 3 (1),11-20. DOI: 10.1007/BF00121385

Grue C.E., Powell G.V.N. y Gladson N.L. (1981). Brain cholinesterase (ChE) activity in nesting starlings: implications for monitoring exposure of nesting songbirds to ChE inhibitors. Bull. Environ. Contam. Toxicol. 26, 544-547. DOI: 10.1007/BF01622134

Hatch R.C. (1988). Toxicología veterinaria. En: Farmacología y terapéutica veterinaria (N.H. Booth y L.E. McDonald, Eds). Acribia, Zaragoza, España, Vol 2, pp. 283-448.

Hill E.F. (1994). Organophosphorus and carbamate pesticides. En: Wildlife toxicology and population modeling. A special publication of SETAC (R.J. Kendall and T.E. Lacher, Jr., Eds.). CRC Press Inc., Florida, EUA, pp. 243-273.

Hill E.F. y Fleming W.J. (1982). Anticholinesterase poisoning of birds: field monitoring and diagnosis of an acute poisoning. Environ. Toxicol. Chem. 1, 27-38. DOI: $10.1002 /$ etc.5620010105

Hooper M.J., Detrich P.J., Weisskopf C.P. y Wilson B.W. (1989). Organophosphorus insecticide exposure in hawks in habiting orchards during winter dormantpraing. Bull. Environ. Contam. Toxicol. 42, 651-659. DOI: $10.1007 / \mathrm{BF} 01700383$

IARC (2015). Evaluation of five organophosphate insecticides and herbicides. International Agency for Research on Cancer. Monographs Vol. 12. World Health Organization.

DOI: $10.1016 / \mathrm{S} 1470-2045(15) 70134-8$

INEGI (2017). México en cifras. Instituto Nacional de Estadística, Geografía e Informática. [en línea]. http:// www.beta.inegi.org. $\mathrm{mx} / \mathrm{app} /$ areasgeograficas/?ag $=00$ $10 / 01 / 2017$

Jeyaratnam J. y Maroni M. (1994). Organophosphorus compounds. Toxicology 91 (1), 15-27. DOI: $10.1016 / 0300-483 X(94) 90236-4$

Lari L., Massi A., Fossi M.C., Casini S., Leonzio C. y Focardi S. (1994). Evaluation of toxic effects of the organophosphorus insecticide Azinphos-Methyl in 
experimentally and naturally exposed birds. Arch. Environ. Contam. Toxicol. 26 (2), 234-239.

DOI: $10.1007 / \mathrm{BF} 00224810$

Maia A.R., Pérez L.M. y Soler R.F. (2012). Comparación de tres métodos de determinación de la actividad colinesterasa plasmática en perro. Rev. Toxicol. 29, 135-140.

Murcia M. A. y Stashenko E. (2008). Determinación de plaguicidas organofosforados en vegetales producidos en Colombia. Agro sur 36 (2), 71-81.

DOI:10.4206/agrosur.2008.v36n2-03

Niethammer K.R. y Baskettt T.S. (1983). Cholinesterase inhibition of birds in habiting wheat field treated whit methyl, parathion and toxaphene. Arch. Environ. Contam. Toxicol. 12 (4), 471-465.

DOI: $10.1007 / \mathrm{BF} 01057591$

Palacios A.A. y Campos Ch. C. (2010). Determinación por HPLC de residuos de insecticida órganofosforado (Methamidophos) en tomates comercializados en Lima Perú. Tesis de Licenciatura. Facultad de Farmacia y Bioquímica, Universidad Nacional Mayor de San Marcos, Lima, Perú, 88 pp.
Ponce G., Cantú P.C., Flores A., Badii M., Zapata R., López B. y Fernández I. (2006). Modo de acción de los insecticidas. Revista Salud Pública y Nutrición 7 (4), 1-18.

Sarwar M. (2015). The danger of pesticides associated whit public health and preventing of the risks. Int. J. Bioinform. Biomed. Eng. 1 (2), 130-136.

Varea M.C., Masoero C., Gentile N., Bosch, B. y Aiassa D. (2003). Biomarcadores posibles para evaluar la exposición laboral a plaguicidas. Revista de Toxicología en línea 13-24.

Vega S. (1985). Evaluación epidemiológica de riesgos causados por agentes químicos ambientales. Toxicología I. Centro Panamericano de Ecología Humana y Salud, Organización Panamericana de la Salud, Organización Mundial de la Salud. México, 69 pp.

Weiss B., Amler S. y Amler R.W. (2004). Pesticides. Pediatrics. 113 (4), 1030-1036.

Whittaker M. (1974) Cholinesterase. En: Methods of ezymatic analysis. 3a ed. (H.U. Bergmeyer, Ed.). Verlag Chemie, Weinheim, Alemania, pp. 52-74.

DOI: $10.1002 /$ star. 19630150713 